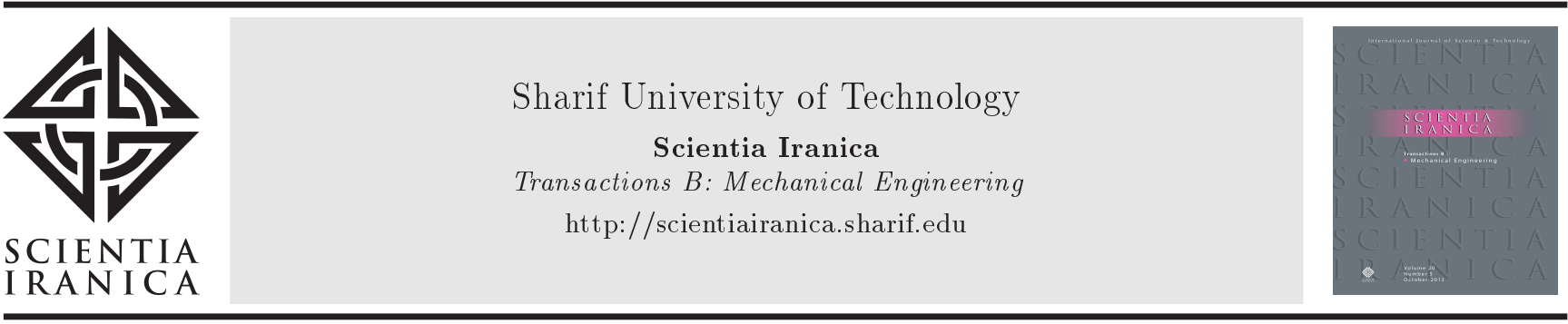

Research Note

\title{
A local differential transform approach to the cubic nonlinear Duffing oscillator with damping term
}

\author{
H. Tunc and M. Sari* \\ Department of Mathematics, Faculty of Arts and Science, Yildiz Technical University, Istanbul 34220, Turkey.
}

Received 24 July 2017; received in revised form 30 October 2017; accepted 13 January 2018

\section{KEYWORDS}

Duffing oscillator;

Differential transform;

Nonlinear behaviour;

Initial value problem;

Exact solution;

Runge-Kutta method.

\begin{abstract}
Nonlinear behaviour of various problems can be described by the Duffing model interpreted as a forced oscillator with a spring, which has a restoring force. In this paper, a new numerical approximation technique based on the differential transform method is introduced for the nonlinear cubic Duffing equation with and without damping effect. Since exact solutions to the corresponding equation for all initial guesses do not exist in the literature, an exact solution is produced first for specific parameters using the Kudryashov method to measure the accuracy of the suggested method. The innovative approach is compared with the semi-analytic differential transform and the fourth-order Runge-Kutta methods. Although the semi-analytic differential transform method is valid only at small-time intervals, it is proved that the innovative approach has the ability to capture nonlinear behaviour of the process even at long-time intervals. The computations indicate that the present technique produces more accurate and computationally more economic results than the rival methods do.
\end{abstract}

(C) 2019 Sharif University of Technology. All rights reserved.

\section{Introduction}

Nonlinear oscillation problems are of immense importance in a broad range of science fields. Duffing oscillators represent one of the most important nonlinear oscillation problems and are described by nonlinear differential equations. The Duffing oscillators have various applied areas, such as modelling of free vibrations of a restrained uniform beam with intermediate lumped mass [1,2], magneto-elastic mechanical systems [3], fluid flow-induced vibration [4], nonlinear dynamics of slender elasticity [5], etc.

Due to the existence of these nonlinearities, the investigation of accurate solutions of the Duffing oscillators is of high importance. Various versions of

\footnotetext{
*. Corresponding author. Tel.: +90212 3834360
} E-mail address: sarim@yildiz.edu.tr (M. Sari). the approximate solution techniques have been used to find solutions to the nonlinear and conservative Duffing equation [6-13]. The homotopy analysis method [6], harmonic balance method [7], the homotopy Padé technique [8], energy balance method [9], coupled homotopy variational approach [10], the Newton harmonic balance method [11], parameter-expanding and max-min approach [12,13], coupling of energy and harmonic balance method [14], Jacobi elliptic functions [15], and parameter-based perturbation technique [16] have all been used to solve a nonlinear Duffing equation without damping effect. If the Duffing oscillator involves the damping effect, the amplitude of the oscillation decreases with time; then, a non-conservative system can be obtained. Most analytical methods do not have the ability to handle the non-conservative Duffing equation. Nevertheless, the Laplace decomposition method [17], homotopy perturbation method [18], Modified Differential Transformation Method (MDTM) [19], and Renormalization 
Group Method (RGM) [20] are capable of solving nonconservative systems.

The DTM was first presented by Zhou [21], and the method was then used to solve differential equations occurred in electric circuit analysis. The DTM has been extensively studied by various researchers for obtaining approximate solutions to various problems of science. This method has been studied to solve system of differential equations [22], fluid flow problems [23,24], magnetohydrodynamics (MHD) boundary-layer equations [25], nonlinear partial differential equations $[26,27]$, and the Duffing oscillator equation with damping effects [19]. In [19,22-28], among a large family of papers, the DTM has become of great help to find an approximate solution in a finite series form about initial time. Generally, the application of this semi-analytic approach leads to accurate and acceptable solutions only about the initial position due to the natural structure of the Taylor series. In [19,25-27], the MDTM has been developed to do away with this disadvantage of the DTM. In the concept of the MDTM, Laplace transformation and Padé approximation are applied, via differential transform method in a global sense, to produce the solution. Even though the MDTM has the ability to decrease numerical error of the approximate solution, one requires more symbolic calculations and more computational cost than the DTM. The DTM can be also used in a local sense to obtain discrete or continuous solutions to differential equations, first introduced by Jang et al. [29].

In this study, an explicit and highly accurate numerical method has been developed by applying the semi-analytic DTM to the equally sized subintervals. The main advantage of the Local Differential Transform Method (LDTM) is that the accuracy of the results can increase with and without a change in the order of the Taylor expansion. Thus, the LDTM has less symbolic calculations than the MDTM does and is more accurate than the DTM and MDTM. One of the significant advantages of the LDTM is that providing high accuracy not only at the neighbourhood of the initial position, but also in the entire domain. Even though the LDTM is similar to the Taylor series method, the computational structure and symbolic calculation are different from both methods. The explicit structure of the LDTM is similar to the fourthorder Runge-Kutta method; however, the LDTM is more flexible and accurate than the RK4 due to the free choices of the Taylor expansion order. By providing a sufficiently small time increment in the LDTM, higherorder accuracy is guaranteed as long as the exact solution is considered to be sufficiently smooth. As shown, in the rest of the paper, the LDTM is suitable for many types of differential equations, even for the differential equations involving power nonlinearities.
Thus, the LDTM has also high capability to solve nonlinear differential equations encountered in various fields of science.

The Kudryashov method [30,31] based on the logistic function and solution of the first-order Riccati equation was used to find exact solutions to the differential equation. The present study produces an exact solution to the cubic nonlinear Duffing equation with specific parameters and initial guesses. The similarity between the first-order Riccati equation and the cubic nonlinear Duffing equation is found herein, and the exact solution to the equation is given in terms of the logistic function. Thus, a special Duffing equation is obtained which is satisfied by the logistic function. To measure the accuracy and stability of the present numerical approach, the LDTM, it is compared with the currently produced exact solution.

In this study, it is proved that the order of the LDTM is stable with increasing time. The cubic nonlinear Duffing equation is considered for various problem parameters including conservative and nonconservative systems. The accuracy of the LDTM method is first compared with the currently produced exact solution and, then, with the DTM and the fourth-order Runge-Kutta method. As demonstrated, the LDTM provides far better results than the other techniques do.

\section{Differential transformation}

The main concept of the differential transform method can be found in the literature [32]. The definitions are reorganized by considering its local sense as follows:

Definition 1. Let $x(t)$ be analytic in domain $T$, and function $\varphi(t, k)$ can be defined as follows:

$$
\frac{d^{k} x(t)}{d t^{k}}=\varphi(t, k) \quad \text { for all } t \in T
$$

where $k$ is a non-negative integer. With the use of Definition 1, the differential transform of function $x(t)$ at any time $t=t_{i}$ in the time domain is locally defined as follows:

$$
X_{i}(k)=\frac{\varphi\left(t_{i}, k\right)}{k !}=\frac{1}{k !}\left[\frac{d^{k} x(t)}{d t^{k}}\right]_{t=t_{i}} .
$$

Definition 2. If $x(t)$ is analytic in domain $T=$ $\left[0, t_{F}\right]$, then $x(t)$ can be denoted by Taylor series at time $t=t_{i}$ by considering Definition 2 as follows:

$$
x(t)=\sum_{k=0}^{\infty}\left(t-t_{i}\right)^{k} X_{i}(k)=D^{-1} X(k),
$$

where $D^{-1}$ denotes an inverse differential transform operator. Function $x(t)$ can be expressed in a finite 
series form with a truncation error of order $(N+1)$ as follows:

$$
\left.x(t)=\sum_{k=0}^{N}\left(t-t_{i}\right)^{k} X(k)+O\left(\left(t-t_{i}\right)\right)^{N+1}\right) .
$$

Notice that the differential transformation of the derivative of any function can be written in terms of the differential transform of the function itself. In virtue of this respect, the differential transformation can be applied to the differential equations. In the literature [32], transformed forms of some well-known functions can be found in detail. By considering the definition of the differential transformation, Table 1 can be generated.

\section{Localized differential transform method for the nonlinear Duffing oscillator}

The following cubic nonlinear Duffing equation can be considered with damping effect:

$$
x^{\prime \prime}+\alpha x^{\prime}+\beta x+\gamma x^{3}=0 \quad \text { for } \quad 0 \leq t \leq t_{f},
$$

with the initial position and velocity:

$$
x(0)=x_{0} \quad \text { and } \quad x^{\prime}(0)=x_{0}^{*},
$$

where $\alpha, \beta$, and $\gamma$ are given constant coefficients. By using $u_{1}(t)=x(t)$ and $u_{2}(t)=x^{\prime}(t)$, Eq. (5) can be transformed into the following system of differential equations:

$$
\begin{aligned}
& u_{1}{ }^{\prime}(t)=u_{2}(t), \\
& u_{2}{ }^{\prime}(t)=-\alpha u_{2}(t)-\beta u_{1}(t)-\gamma u_{1}^{3}(t) .
\end{aligned}
$$

Considering differential transformation of Eqs. (7) and (8) leads to:

$$
\mathbf{U}(k+1)=\frac{1}{k+1}\left(A \mathbf{U}(k)+B_{k}\right),
$$

where $\mathbf{U}(k)=\left[U_{1}(k), U_{2}(k)\right]^{T}$ is the differential transformation of:

$$
\begin{aligned}
& {\left[u_{1}(t), u_{2}(t)\right]^{T},} \\
& B_{K}=\left[0,-\gamma \sum_{l=0}^{k} \sum_{n=0}^{l} U_{2}(n) U_{2}(l-n) U_{2}(k-l)\right]^{T} .
\end{aligned}
$$

Matrix $A$ can be defined as follows:

$$
A=\left[\begin{array}{cc}
0 & 1 \\
-\beta & -\alpha
\end{array}\right] \text {. }
$$

The interval $\left[0, t_{f}\right]$ is partitioned into $N$ subdomains with equally spaced grid points defined as $0=t_{0}<$ $t_{1}<\ldots<t_{N}=t_{f}$ such that $t_{i+1}=t_{i}+d t$ where $d t=\frac{t_{f}}{N}$. For the first subinterval, function $\mathbf{u}(t)$ can be approximated by $\mathbf{u}_{0}(t)$ such that:

$$
\begin{aligned}
& \mathbf{u}_{0}(t)=\mathbf{U}_{0}(0)+\mathbf{U}_{0}(1) t+\mathbf{U}_{0}(2) t^{2}+\ldots+\mathbf{U}_{0}(K) t^{K}, \\
& \mathbf{U}_{0}(k+1)=\frac{1}{k+1}\left(A \mathbf{U}_{0}(k)+B_{k}\right),
\end{aligned}
$$

where $\mathbf{U}_{0}(0)=\left[x_{0}, x_{0}^{*}\right]^{T}$ is the initial guess, and $K$ is the order of the differential transformation. Approximate value of the required function $\mathbf{u}(t)$ at $t=t_{1}$ can be evaluated as follows:

$$
\mathbf{u}\left(t_{1}\right) \cong \mathbf{u}_{0}\left(t_{1}\right)=\sum_{k=0}^{K} \mathbf{U}_{0}(k) d t^{k} .
$$

The main idea for building up the LDTM is that the obtained solution is taken to be the initial value of the next iteration, namely $\mathbf{u}_{1}\left(t_{1}\right)=\mathbf{U}_{1}(0)=\mathbf{u}_{0}\left(t_{1}\right)$. Thus, the approximate solution $\mathbf{u}_{1}(t)$ for the second subdomain can be expressed as follows:

$$
\begin{aligned}
\mathbf{u}_{1}(t)= & \mathbf{U}_{1}(0)+\mathbf{U}_{1}(1)\left(t-t_{1}\right)+\mathbf{U}_{1}(2)\left(t-t_{1}\right)^{2} \\
& +\ldots+\mathbf{U}_{1}(K)\left(t-t_{1}\right)^{K}
\end{aligned}
$$

Table 1. Algebraic properties of differential transformation and transformations of well-known functions.

\begin{tabular}{ll}
\hline \multicolumn{1}{c}{ Function } & Transformed function \\
\hline$X(t)=y(t) \pm z(t)$ & $X(k)=Y(k) \pm Z(k)$ \\
$x(t)=\alpha y(t)$ & $X(k)=\alpha Y(k)$ \\
$x(t)=\frac{d y(t)}{d t}$ & $X(k)=(k+1) Y(k+1)$ \\
$x(t)=\frac{d^{m} y(t)}{d t^{m}}$ & $X(k)=(k+1)(k+2) \ldots(k+m) Y(k+m)$ \\
$x(t)=t^{m}$ & $X(k)= \begin{cases}1 & \text { if } k=m \\
C(m, k) t_{k}^{(k-m)} & \text { if } k \neq m\end{cases}$ \\
$x(t)=\exp (\gamma t)$ & $X(k)=\exp \left(\gamma t_{k}\right) \frac{\gamma^{k}}{k !}$ \\
$x(t)=y(t) z(t)$ & $X(k)=\sum_{l=0}^{k} Y(l) Z(k-l)$ \\
$x(t)=y^{m}(t)$ & $X(k)=\sum_{l=0}^{k} Y^{m-1}(l) Y(k-l)$ \\
\hline
\end{tabular}


Coefficients $\mathbf{U}_{1}(i)$ of function $\mathbf{u}_{1}(t)$ can be found using the same recursive relation in Eq. (12) with the initial condition $\mathbf{U}_{1}(0)=\mathbf{u}_{0}\left(t_{1}\right)$. Then, approximate solution $\mathbf{u}\left(t_{2}\right)$ is written as follows:

$$
\mathbf{u}\left(t_{2}\right) \cong \mathbf{u}_{1}\left(t_{2}\right)=\sum_{k=0}^{K} \mathbf{U}_{1}(k) d t^{k}
$$

Hence, the approximate solution at grid point $t_{i+1}$ can be stated as follows:

$$
\mathbf{u}\left(t_{i+1}\right) \cong \mathbf{u}_{i}\left(t_{i+1}\right)=\sum_{k=0}^{K} \mathbf{U}_{i}(k) d t^{k}
$$

where subscripts are taken to be $i=0,1,2, \ldots, N-1$.

\section{Kudryashov method}

The Kudryashov method [30,31] is an analytic technique for obtaining exact solutions to differential equations using the following logistic function:

$$
Q(t)=\frac{1}{1+e^{-t}}
$$

where function $Q(t)$ is a particular solution to the firstorder Riccati equation:

$$
Q_{t}-Q+Q^{2}=0
$$

Differentiating Eq. (18) with respect to $t$ and replacing the term $Q_{t}$ by $Q-Q^{2}$ leads to the following nonlinear differential equation:

$$
\begin{gathered}
Q_{t t}-Q_{t}+2 Q^{2}-2 Q^{3}+F\left(Q, Q_{t}, \ldots\right) \\
\left(Q_{t}-Q+Q^{2}\right)=0
\end{gathered}
$$

where $F\left(Q, Q_{t}, \ldots\right)$ is an arbitrary function of function $Q(t)$ and its derivatives. Taking $F\left(Q, Q_{t}, \ldots\right)=-2$ to vanish quadratic term yields:

$$
Q_{t t}-3 Q_{t}+2 Q-2 Q^{3}=0
$$

As clearly seen, Eq. (20) is a cubic nonlinear Duffing equation (5) with the following parameter values:

$$
\alpha=-3, \beta=2, \gamma=-2, x_{0}=0.5, x_{0}^{*}=0.25
$$

Thus, logistic function (17) is an exact solution to the cubic nonlinear Duffing equation (20). However, at long-time interval, the logistic function (17) cannot represent the physical behavior of the Duffing equation since function (17) is not harmonic. To measure the effectiveness of the LDTM, solution (17) is used as a test case and without considering the physical behavior of the logistic function.

\section{Numerical experiments}

This section is devoted to the numerical illustration of various test problems including conservative and non-conservative systems. Since the exact solution to the cubic nonlinear Duffing equation does not exist, the currently produced exact solution (17) is first considered.

In later cases, physically acceptable parameters and the nonlinear oscillations are assumed that are demonstrated at long-time intervals.

For the last three cases, it is preferred to use high values of $N$, and the obtained results are accepted as exact solutions to evaluate error norms [33]. Accuracy and stability of the obtained results are figured out by demonstrating error norms and order of the present method. The produced results are compared with the RK4 solutions and the DTM solutions.

Case 1: In this case, the cubic nonlinear Duffing equation is considered with parameters (21) and exact solution (17). For the exact solution, DTM and LDTM are compared in Figure 1 at the interval $t \in[0,5]$. For the present algorithm, parameters $K=10$ and $N=100$ are used. In Figure 2, the relative and absolute errors of the LDTM and RK4 are compared with the same parameters used in Figure 1. As seen in the figures, the LDTM produces more acceptable results than the other two techniques.

Case 2 [19]: In the present case, low damping effect, strong nonlinearity, and initial displacement are considered with the following parameters:

$$
\alpha=0.5, \quad \beta=\gamma=25, \quad x_{0}=0.1, \quad x_{0}^{*}=0 .
$$

In Figure 3, the present numerical technique is compared with the DTM given in the literature [19]. As seen in Figure 3, the DTM produces physically

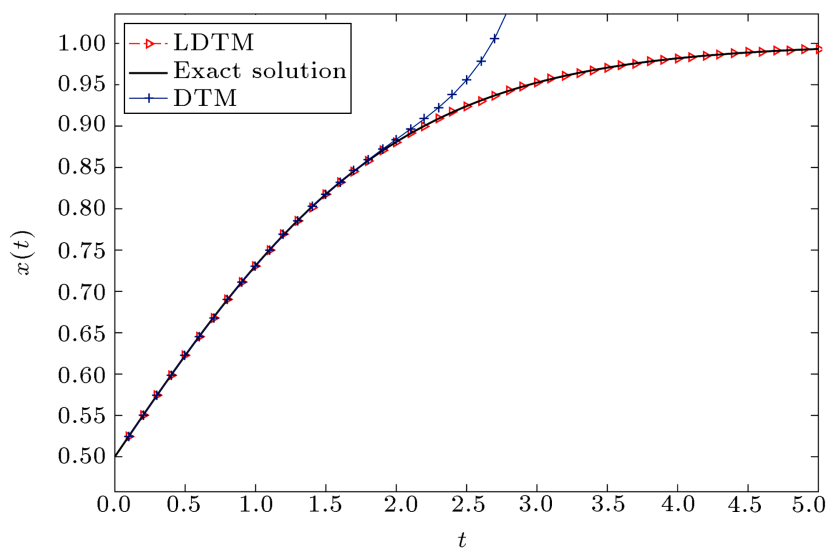

Figure 1. Comparison of the LDTM and DTM solutions with the exact solution for $\alpha=-3, \beta=2, \gamma=-2$, $x_{0}=0.5$, and $x_{0}^{*}=0.25$. 

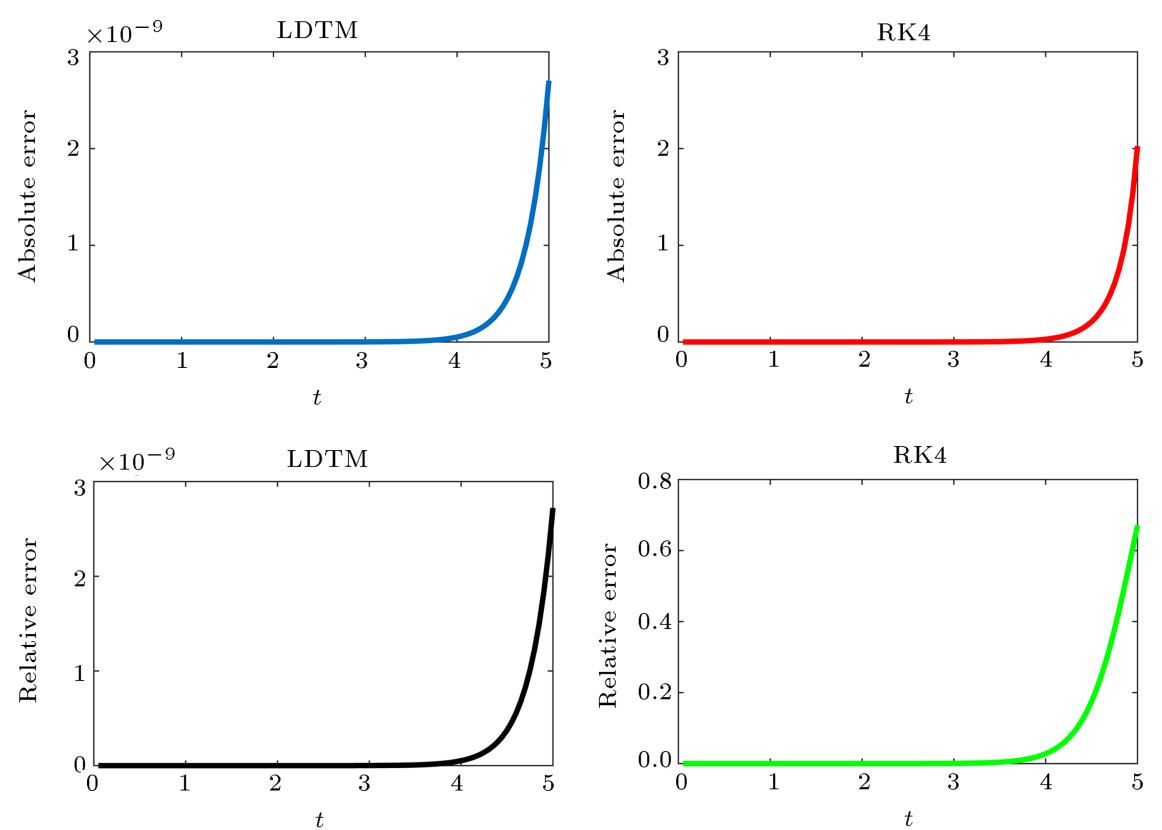

Figure 2. Comparison of the LDTM with the RK4 in terms of absolute and relative errors produced for $\alpha=-3, \beta=2$, $\gamma=-2, x_{0}=0.5$, and $x_{0}^{*}=0.25$.

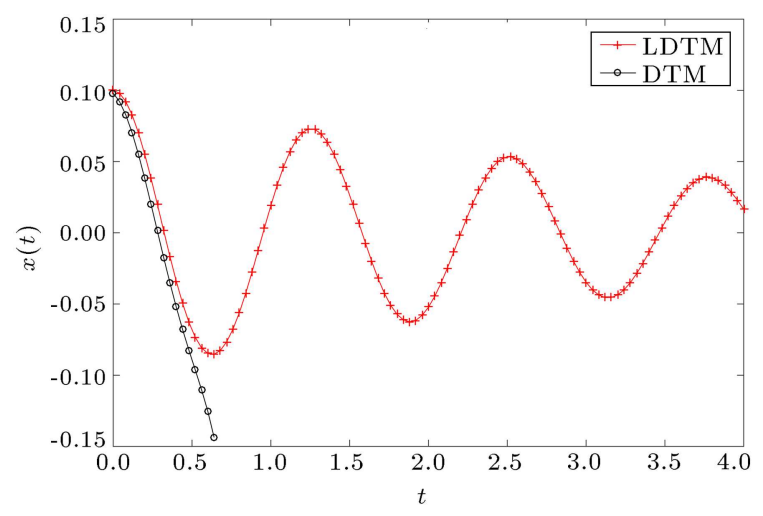

Figure 3. The LDTM and DTM solutions for $\alpha=0.5$, $\beta=\gamma=25, x_{0}=0.1$, and $x_{0}^{*}=0$.

acceptable results only at the small-time interval. However, the non-conservative system represented by the Duffing equation is exactly captured by the LDTM. Parameters $K=7$ and $N=100$ are used both for the LDTM and DTM in Figure 3. In Table 2, the computed solutions are compared for various values of parameters $N$ and $K=10$. The reference solution can be considered as an exact solution with parameters $N=1000$ and $K=10$.

Case 3 [23]: In this case, critical damping, strong nonlinearity, and initial displacement in the cubic nonlinear Duffing equation (5) can be assumed with the following parameters:

$$
\alpha=2, \quad \beta=1, \quad \gamma=25, \quad x_{0}=0.1, \quad x_{0}^{*}=0 .
$$

The comparison of the solutions produced both by the LDTM and DTM for the non-conservative Duffing equation with parameters (18) is demonstrated in Figure 4. The DTM solutions are in agreement with the LDTM solutions only at the small-time interval. In Figure 4, parameters $K=7$ and $N=100$ are used in both cases. The absolute errors produced by the LDTM with various values of $K$ are demonstrated in Table 3 by considering the solution produced for parameters $N=1000$ and $K=10$ as reference solutions.

Case 4: In the last case, the conservative case of the cubic nonlinear Duffing equation can be assumed with the following parameter values:

$$
\alpha=0, \quad \beta=\gamma=25, \quad x_{0}=0.1, \quad x_{0}^{*}=0 .
$$

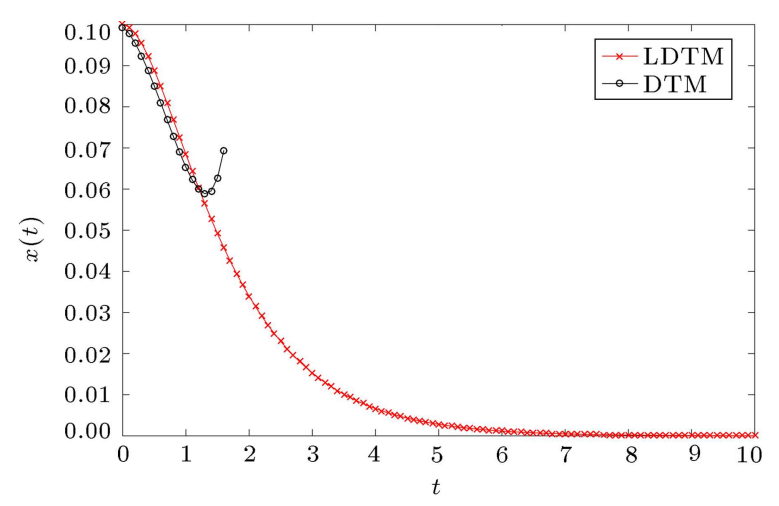

Figure 4. The LDTM and DTM solutions for $\alpha=2$, $\beta=1, \gamma=25, x_{0}=0.1$, and $x_{0}^{*}=0$. 
Table 2. Comparison of the LDTM solutions for various parameters values $N$ with $K=10$.

\begin{tabular}{cccccc}
\hline $\boldsymbol{t}$ & $\boldsymbol{N}=\mathbf{2 0}$ & $\boldsymbol{N}=\mathbf{4 0}$ & $\boldsymbol{N}=\mathbf{6 0}$ & $\boldsymbol{N}=\mathbf{8 0}$ & $\boldsymbol{N}=\mathbf{1 0 0}$ \\
\hline 0.5 & $2.96 \mathrm{E}-06$ & $4.27 \mathrm{E}-10$ & $1.48 \mathrm{E}-11$ & $9.50 \mathrm{E}-13$ & $1.07 \mathrm{E}-13$ \\
1.0 & $3.29 \mathrm{E}-06$ & $9.69 \mathrm{E}-10$ & $3.82 \mathrm{E}-12$ & $1.19 \mathrm{E}-13$ & $3.30 \mathrm{E}-14$ \\
2.0 & $7.50 \mathrm{E}-07$ & $5.59 \mathrm{E}-10$ & $1.16 \mathrm{E}-11$ & $6.61 \mathrm{E}-13$ & $7.03 \mathrm{E}-14$ \\
3.0 & $1.80 \mathrm{E}-06$ & $1.86 \mathrm{E}-10$ & $2.43 \mathrm{E}-12$ & $2.63 \mathrm{E}-13$ & $3.52 \mathrm{E}-14$ \\
4.0 & $5.12 \mathrm{E}-07$ & $6.90 \mathrm{E}-10$ & $9.66 \mathrm{E}-12$ & $4.61 \mathrm{E}-13$ & $4.36 \mathrm{E}-14$ \\
5.0 & $8.46 \mathrm{E}-07$ & $6.56 \mathrm{E}-11$ & $3.74 \mathrm{E}-12$ & $2.63 \mathrm{E}-13$ & $3.08 \mathrm{E}-14$ \\
\hline
\end{tabular}

Table 3. Comparison of the LDTM solutions for various parameters values $K$ with $N=100$.

\begin{tabular}{cccccc}
\hline $\boldsymbol{t}$ & $\boldsymbol{K}=\mathbf{2}$ & $\boldsymbol{K}=\mathbf{4}$ & $\boldsymbol{K}=\mathbf{6}$ & $\boldsymbol{K}=\mathbf{8}$ & $\boldsymbol{K}=\mathbf{1 0}$ \\
\hline 0.5 & $2.80 \mathrm{E}-05$ & $3.35 \mathrm{E}-09$ & $2.07 \mathrm{E}-12$ & $2.75 \mathrm{E}-15$ & $4.44 \mathrm{E}-16$ \\
1.0 & $2.10 \mathrm{E}-05$ & $8.85 \mathrm{E}-09$ & $6.30 \mathrm{E}-12$ & $1.87 \mathrm{E}-15$ & $4.02 \mathrm{E}-16$ \\
2.0 & $6.19 \mathrm{E}-06$ & $7.50 \mathrm{E}-09$ & $3.79 \mathrm{E}-12$ & $8.33 \mathrm{E}-17$ & $5.55 \mathrm{E}-17$ \\
3.0 & $1.27 \mathrm{E}-05$ & $2.57 \mathrm{E}-09$ & $1.69 \mathrm{E}-12$ & $3.12 \mathrm{E}-17$ & $1.01 \mathrm{E}-16$ \\
4.0 & $9.60 \mathrm{E}-06$ & $5.21 \mathrm{E}-10$ & $7.80 \mathrm{E}-13$ & $5.03 \mathrm{E}-17$ & $6.85 \mathrm{E}-17$ \\
5.0 & $5.68 \mathrm{E}-06$ & $2.85 \mathrm{E}-11$ & $3.53 \mathrm{E}-13$ & $3.82 \mathrm{E}-17$ & $3.08 \mathrm{E}-17$ \\
\hline
\end{tabular}

As seen in Figure 5, the LDTM produces physically acceptable results, while the DTM solutions are valid only about the initial time. The period of the produced solution is obtained about $t_{p}=2.5$. The results in Figure 5 are evaluated to select the parameters to be $K=7$ and $N=100$. The absolute errors of the

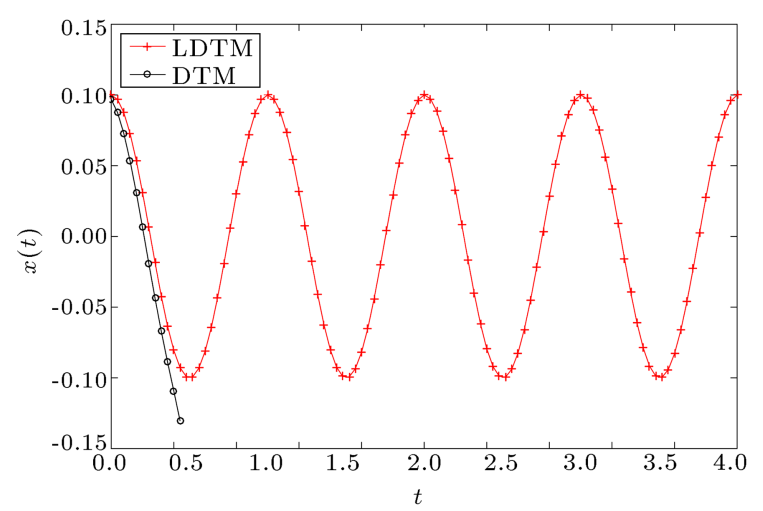

Figure 5. Comparison of the LDTM with DTM solutions for $\alpha=0, \beta=\gamma=25, x_{0}=0.1$, and $x_{0}^{*}=0$. present algorithm with various values of $K$ are shown in Table 4. The present solution produced for parameters $N=1000$ and $K=10$ is accepted as reference solution in Table 4.

\section{Conclusions and recommendation}

The cubic nonlinear Duffing equation representing behaviour of forced oscillators with a spring, which has a restoring force, has been solved by a local differential transformation method. Since the exact solutions of the corresponding equation for all initial guesses do not exist in the literature, an exact solution for specific parameters was first produced using the Kudryashov method to measure the accuracy of the suggested method. In addition, the derivation of the exact solution to the Duffing equation can be accepted to be an important consequence. It has been shown that the LDTM produces highly accurate results in comparison to its rivals. It has been proved that the innovative approach has great ability to capture non-

Table 4. Comparison of the LDTM solutions for various parameters values $K$ with $N=100$.

\begin{tabular}{cccccc}
\hline $\boldsymbol{t}$ & $\boldsymbol{K}=\mathbf{2}$ & $\boldsymbol{K}=\mathbf{4}$ & $\boldsymbol{K}=\mathbf{6}$ & $\boldsymbol{K}=\mathbf{8}$ & $\boldsymbol{K}=\mathbf{1 0}$ \\
\hline 1.0 & $2.18 \mathrm{E}-02$ & $2.67 \mathrm{E}-04$ & $1.74 \mathrm{E}-06$ & $1.12 \mathrm{E}-08$ & $1.45 \mathrm{E}-10$ \\
2.0 & $1.93 \mathrm{E}-02$ & $1.07 \mathrm{E}-04$ & $6.43 \mathrm{E}-07$ & $3.73 \mathrm{E}-09$ & $4.34 \mathrm{E}-11$ \\
4.0 & $9.45 \mathrm{E}-02$ & $7.95 \mathrm{E}-04$ & $5.02 \mathrm{E}-06$ & $2.73 \mathrm{E}-08$ & $2.77 \mathrm{E}-10$ \\
6.0 & $1.34 \mathrm{E}-01$ & $1.63 \mathrm{E}-03$ & $9.98 \mathrm{E}-06$ & $4.05 \mathrm{E}-08$ & $2.22 \mathrm{E}-10$ \\
8.0 & $2.12 \mathrm{E}-02$ & $1.97 \mathrm{E}-03$ & $1.19 \mathrm{E}-05$ & $3.91 \mathrm{E}-08$ & $4.48 \mathrm{E}-11$ \\
10.0 & $2.21 \mathrm{E}-01$ & $1.30 \mathrm{E}-03$ & $7.94 \mathrm{E}-06$ & $2.86 \mathrm{E}-08$ & $6.92 \mathrm{E}-11$ \\
\hline
\end{tabular}


linear behaviour of the physical process even at longtime intervals as opposed to the compared methods, which are effective mostly at short-time intervals. Note that the suggested approach has been seen to be a very good alternative to achieving a high degree of accuracy while analysing the processes represented by the model equation. Future studies can focus on the design of the current technique to physical processes represented by more involved nonlinear models with the forcing terms.

\section{References}

1. Ahmadian, M.T., Mojahedi, M., and Moeenfard, H. "Free vibration analysis of a nonlinear beam using homotopy and modified Lindstedt-Poincare methods", Journal of Solid Mechanics, 1, pp. 29-36 (2009).

2. Bakhtiari-Nejad, F. and Nazari, M. "Nonlinear vibration analysis of isotropic cantilever plate with viscoelastic laminate", Nonlinear Dynam., 56, pp. 325356 (2009).

3. Guckenheimer, J. and Holmes, P., Nonlinear Oscillations, Dynamical Systems and Bifurcations of Vector Fields, Springer-Verlag, New York, USA (1983).

4. Srinil, N. and Zanganeh, H. "Modelling of coupled cross-flow/in-line vortex-induced vibrations, using double Duffing and Van der Pol oscillators", Ocean Sci. J., 53, pp. 83-97 (2012).

5. Guo, Z., Leung, A.Y.T., and Yang, H.X. "Iterative homotopy harmonic balancing approach for conservative oscillator with strong odd-nonlinearity", Appl. Math. Model., 35, pp. 1717-1728 (2011).

6. Liao, S.J. and Cheung, A.T. "Application of homotopy analysis method in nonlinear oscillations", J. Appl. Mech., 65, pp. 914-922 (1998).

7. Mickens, R.E. "Mathematical and numerical study of the Duffing-harmonic oscillator", J. Sound Vib., 244, pp. 563-567 (2001).

8. Pirbodaghi, P., Hoseini, S.H., Ahmadian, M.T., and Farrahi, G.H. "Duffing equations with cubic and quantic nonlinearities", Comput. Math. Appl., 57, pp. 500506 (2009).

9. Ganji, D.D., Gorji, M., Soleimani, S., and Esmaelpour, M. "Solution of nonlinear cubic-quintic Duffing oscillators using He's energy balance method", J. Zhejiang Univ-Sc. B, 10, pp. 1263-1268 (2009).

10. Khan, Y., Akbarzade, M., and Kargar, A. "Coupling of homotopy and the variational approach for a conservative oscillator with strong odd-nonlinearity", Sci. Iran., 19, pp. 417-422 (2012).

11. Joubari, M.M., Asghari, R., and Jahromy, M.Z. "Investigation of the dynamic behavior of periodic systems with Newton harmonic balance method", Math. Comput. Model., 44, pp. 418-427 (2012).

12. Zengin, F.O., Kaya, M.O., and Demirbag, S.A. "Approximate period calculation for some strongly nonlinear oscillation by He's parameter-expanding methods", Nonlinear Anal. Real., 10, pp. 2177-2182 (2009).
13. Ibsen, L.B., Barari, A., and Kimiaeifar, A. "Analysis of highly nonlinear oscillation systems using He's maxmin method and comparison with homotopy analysis and energy balance methods", Sadhana-Acad. P. Eng. S., 35, pp. 433-448 (2010).

14. Razzak, M.A. "Coupling of energy and harmonic balance method for solving a conservative oscillator with strong odd-nonlinearity", Sci. Iran., 55, pp. 991998 (2018).

15. Razzak, M.A. "A new analytic approach to investigate the strongly nonlinear oscillators", Alexandria Engineering Journal, 55, pp. 1827-1834 (2016).

16. El-Naggar, A.M. and Ismail, G.M. "Analytical solution of strongly nonlinear Duffing oscillators", Alexandria Engineering Journal, 55, pp. 1581-1585 (2016).

17. Khan, Y. and Austin, F. "Application of the Laplace decomposition method to nonlinear homogenous and non-homogeneous advection equations", Zeitschrift für Naturforschung A, 65a, pp. 849-853 (2010).

18. Khan, Y. and Wu, Q. "Homotopy perturbation transform method for nonlinear equations using He's polynomials", Comput. Math. Appl., 61, pp. 1963-1967 (2011).

19. Nourazar, S. and Mirzabeigy, A. "Approximate solution for nonlinear Duffing oscillator with damping effect using the modified differential transform method", Sci. Iran., 20, pp. 364-368 (2013).

20. Hosseini, S.A.A. "Some considerations on higher order approximation of Duffing equation in the case of primary resonance", Sci. Iran., 20, pp. 1464-1473 (2013).

21. Zhou, J.K. Differential Transformation and its Application for Electrical Circuits, Huarjung University Press, Wuuhahn, China (1986).

22. Abdel-Halim Hassan, I.H. "Application to differential transformation method for solving systems of differential equations", Appl. Math. Model., 32, pp. 2552-2559 (2008).

23. Mao, Q. "Design of shaped piezoelectric modal sensors for cantilever beams with intermediate support by using differential transform method", Appl. Acoust., 73, pp. 144-149 (2012).

24. Rashidi, M.M. and Domairry, G. "New analytical solution of the three-dimensional Navier Stokes equations", Mod. Phys. Lett. B, 23, pp. 3147-3155 (2009).

25. Rashidi, M.M. "The modified differential transform method for solving MHD boundary-layer equations", Comput. Phys. Commun., 180, pp. 2210-2217 (2009).

26. Al-Amr, M.O. "New applications of reduced differential transform method", Alexandria Engineering Journal, 53, pp. 243-247 (2004).

27. Taghavi, A., Babaei, A., and Mohammadpour, A. "Application of reduced differential transform method for solving nonlinear reaction-diffusion-convection problems", Applications \& Applied Mathematics, 10, pp. 162-170 (2015). 
28. Hesam, S., Nazemi, A.N., and Haghbin, A. "Analytical solution for the generalized Kuratomo-Sivashinsky equation by the differential transform method", Sci. Iran., 20, pp. 1805-1811 (2013).

29. Jang, M.-J., Chen, C.-L., and. Liyb, Y.-C "On solving the initial-value problems using the differential transformation method", Appl. Math. Comput., 115, pp. 145-160 (2000).

30. Kudryashov, N.A. "Logistic function as solution of many differential equations", Appl. Math. Model., 39, pp. 5733-5742 (2015).

31. Kudryashov, N.A. "Polynomials in logistic function and solitary waves of nonlinear differential equations", Appl. Math. Comput., 219, pp. 9245-9253 (2013).

32. Abdel-Halim Hassan, I.H. "Differential transformation technique for solving higher-order initial value problems", Appl. Math. Comput., 154, pp. 299-311 (2004).

33. Pan, X. and Zhang, L. "A new finite difference scheme for the Rosenau-Burgers equation", Appl. Math. Comput., 218, pp. 8917-8924 (2012).

\section{Biographies}

Huseyin Tunc is currently a $\mathrm{PhD}$ student at the Department of Mathematics at Yildiz Technical University. He received the BSc degree from the Ege University in Mathematics in 2015 and the MSc degree in Mathematics from Yildiz Technical University, Turkey in 2017. His research interests include approximate solutions to partial differential equations, fluid dynamics, finite element method, and high-order time integration methods. He has 5 international scientific publications.

Murat Sari is a Professor of Mathematics at Yildiz Technical University, Istanbul, Turkey. He received the BSc degree from the Ondokuzmayis University in 1991, and the MPhil (transferred) and PhD degrees in Mathematics from University of South Wales, UK, in 1997 and 2000, respectively. His current research interests include numerical solutions of differential equations, computational methods, computational fluid dynamics, modelling of nonlinear behavior, economical modelling, and biomechanical/biomedical modelling. He has about 50 high-quality scientific papers, over 40 conference proceedings, and written/translated various chapters in some books. He supervised 12 graduate students and has been supervising 8 graduate students. He has carried out many talks in various universities in different countries such as Petroleum-Gas University of Ploieşti, Romania, Pardubice University, Czech Republic, Université Toulouse III-Paul Sabatier, France, Ningbo University in China, Pamukkale University, Bogazici University, Yildiz Technical University, Erzincan University, Dumlupinar University in Turkey. $\mathrm{He}$ is a reviewer for many international high-quality journals. 